Portland State University

PDXScholar

Electrical and Computer Engineering Faculty

Publications and Presentations

Electrical and Computer Engineering

$12-1-1990$

\title{
Oscillation frequency in high-gain lasers
}

Lee W. Casperson

Portland State University

Follow this and additional works at: https://pdxscholar.library.pdx.edu/ece_fac

Part of the Electrical and Computer Engineering Commons

Let us know how access to this document benefits you.

Citation Details

Casperson, L. W. (1990). Oscillation frequency in high-gain lasers. Physical Review A (General Physics), $42(11) 6721-6731$.

This Article is brought to you for free and open access. It has been accepted for inclusion in Electrical and Computer Engineering Faculty Publications and Presentations by an authorized administrator of PDXScholar. Please contact us if we can make this document more accessible: pdxscholar@pdx.edu. 


\title{
Oscillation frequency in high-gain lasers
}

\author{
Lee W. Casperson* \\ Department of Physics, University of Otago, P. O. Box 56, Dunedin, New Zealand
}

(Received 25 June 1990)

\begin{abstract}
The calculation of the oscillation frequency of a single-mode laser oscillator is examined systematically. Mode-pulling formulas are developed for a variety of laser systems, and the limitations of a standard approximation are explored.
\end{abstract}

\section{INTRODUCTION}

The oscillation frequency of a laser is an important parameter for many applications, and much effort has been expended on techniques for the stabilization or controlled modulation of the laser frequency. A successful understanding of such techniques presupposes that one has a sufficiently accurate method of frequency calculation. The simplest concept of the longitudinal mode frequency being an integer multiple of the speed of light divided by the cavity length is often inadequate because of refraction and more complicated dispersion effects of the laser medium. The purpose of this study has been to systematically reexamine the methods of calculating the oscillation frequency in the simplest class of $\mathrm{cw}$ single-mode one-dimensional lasers. While there would perhaps be some intrinsic value in just reviewing previous modefrequency calculation methods, the emphasis here is on some specific improvements.

The most basic aspects of laser mode calculation were being explored before the first laser was built, ${ }^{1}$ and a standard mode-pulling formula was introduced by Townes in $1961 .^{2}$ The first comprehensive study of mode pulling in homogeneously and inhomogeneously broadened lasers was reported by Bennett, ${ }^{3}$ and the semiclassical framework for most rigorous mode-frequency studies was provided by Lamb. ${ }^{4}$ Under conditions of very severe mode pulling it was also found that more than one frequency can have the same wavelength in a laser oscillator. ${ }^{5}$ The present treatment focuses on improved frequency calculations for the simplest possible ring and standing-wave laser geometries. To increase the practical applicability of the results, the analysis includes a more realistic energy decay scheme than most previous four-level laser models. More fundamentally, it is shown how to avoid an approximation in the field equations that has been employed in previous treatments of laser mode frequency. ${ }^{2-33}$ The approximate equations have provided adequate agreement with experimental data obtained using heliumneon, $3,6-10,12,14,18,20,23,26,31,32$ xenon, ${ }^{5,24}$ hydrogen fluoride, ${ }^{25,28,30}$ iodine, ${ }^{29}$ and probably many other lasers as well. The standard laser frequency-pulling theory has also been applied to the frequency analysis of a gyrotron oscillator, ${ }^{34}$ and other nonlaser oscillator applications are also likely. The errors caused by the field-equation approximation would tend to be greatest for the very short high-gain lasers that have been attracting increased attention recently.

The basic semiclassical formalism for a onedimensional ring laser is developed in Sec. II. Section III includes a detailed analysis of mode pulling in homogeneously broadened lasers with an emphasis on the implications of the approximation mentioned above. All results, exact and approximate, can be obtained analytically for the case of homogeneous broadening. The more difficult case of inhomogeneous broadening is considered in Sec. IV, and the formulation for the various categories of standing-wave lasers is summarized in Sec. V. For lasers with inhomogeneous broadening or general mixed broadening, numerical or graphical techniques are required. The analytic mode-pulling formulas for homogeneously broadened lasers are shown to be the same whether the resonator is of the ring or standing-wave type. Section VI includes a discussion of the practical implications of the new and more exact mode pulling formulas. The Appendix contains a brief derivation of the leading terms in the saturated gain and dispersion formulas for ring lasers that are primarily inhomogeneously broadened.

\section{RING-LASER MODEL}

The focus of this study is on the development of a more exact treatment of the oscillation frequency in simple single-mode laser oscillators. For this purpose the many complicating features that have been included in more specialized treatments of laser oscillation may be largely neglected. Thus, the starting point for this study is a basic one-dimensional semiclassical laser model that includes inhomogeneous broadening and was developed for studies of laser instabilities. ${ }^{35}$ The density-matrix equations in the model take the form

$\left[\frac{\partial}{\partial t}+v \frac{\partial}{\partial z}\right] \rho_{a b}\left(v, \omega_{\alpha}, z, t\right)=-\left(i \omega_{\alpha}+\gamma\right) \rho_{a b}\left(v, \omega_{\alpha}, z, t\right)-\frac{i \mu}{\hbar} E(z, t)\left[\rho_{a a}\left(v, \omega_{\alpha}, z, t\right)-\rho_{b b}\left(v, \omega_{\alpha}, z, t\right)\right]$ 
$\left[\frac{\partial}{\partial t}+v \frac{\partial}{\partial z}\right] \rho_{a a}\left(v, \omega_{\alpha}, z, t\right)=\lambda_{a}\left(v, \omega_{\alpha}, z, t\right)-\gamma_{a} \rho_{a a}\left(v, \omega_{\alpha}, z, t\right)+\left(\frac{i \mu}{\hbar} E(z, t) \rho_{b a}\left(v, \omega_{\alpha}, z, t\right)+\right.$ c.c. $)$,

$\left[\frac{\partial}{\partial t}+v \frac{\partial}{\partial z}\right] \rho_{b b}\left(v, \omega_{\alpha}, z, t\right)=\lambda_{b}\left(v, \omega_{\alpha}, z, t\right)-\gamma_{b} \rho_{b b}\left(v, \omega_{\alpha}, z, t\right)+\gamma_{a b} \rho_{a a}\left(v, \omega_{\alpha}, z, t\right)-\left(\frac{i \mu}{\hbar} E(z, t) \rho_{b a}\left(v, \omega_{\alpha}, z, t\right)+\right.$ c.c. $)$,

$\rho_{b a}\left(v, \omega_{\alpha}, z, t\right)=\rho_{a b}^{*}\left(v, \omega_{\alpha}, z, t\right)$,

where the subscripts $a$ and $b$ denote the upper and lower laser levels, respectively, $\gamma_{a}$ and $\gamma_{b}$ are the total decay rates for these levels, $\gamma_{a b}$ is the rate of direct decays from level $a$ to level $b, \gamma$ is the decay rate for the off-diagonal elements, $\lambda_{a}$ and $\lambda_{b}$ are the pumping rates, $\mu$ is the dipole moment for the laser transition, and the notation c.c. means the complex conjugate of the preceding terms. The laser medium is assumed to have both Doppler and non-Doppler broadening mechanisms, with $v$ being the $z$ component of the velocity and $\omega_{\alpha}$ the center frequency of the laser transition for members of an atomic or molecular class $\alpha$. The decay process represented by $\gamma_{a b}$ is often missing from theoretical studies, but it can have an important effect on the population of the lower level of the laser transition.

To the density-matrix equations for the atomic or molecular populations and polarizations must be added an equation for the electric field. The wave equation for the electric field of a linearly polarized wave in a laser medium can be written

$$
\begin{aligned}
& \frac{\partial^{2} E(z, t)}{\partial z^{2}}-\mu_{1} \sigma \frac{\partial E(z, t)}{\partial t}-\mu_{1} \epsilon_{1} \frac{\partial^{2} E(z, t)}{\partial t^{2}} \\
&=\mu_{1} \frac{\partial^{2} P(z, t)}{\partial t^{2}} .
\end{aligned}
$$

The permeability $\mu_{1}$ and permittivity $\epsilon_{1}$ should be understood to include all of the magnetic and dielectric properties of the laser medium except for the polarization $P$, which is due to the lasing atoms or molecules. The polarization driving this equation can be related back to the off-diagonal density-matrix elements by

$$
P(z, t)=\int_{0}^{\infty} \int_{-\infty}^{\infty} \mu \rho_{a b}\left(v, \omega_{\alpha}, z, t\right) d v d \omega_{\alpha}+\text { c.c. }
$$

Equations (1)-(6) are a complete set from which the time and space dependences of the electric field and of the atomic or molecular parameters can be determined, subject to the boundary conditions at the resonator mirrors.

The simplest solutions of Eqs. (1) - (6) are those that apply to uniformly pumped steady-state one-directional ring lasers, in which both the electric field and the polarization are constant amplitude traveling waves. If the losses in a ring laser can be considered to be uniformly distributed, then the rapid time and space variations in Eqs. (1)-(6) can be factored out by means of the substitutions

$$
E(z, t)=\frac{1}{2} E^{\prime} \exp (i k z-i \omega t)+\text { c.c. },
$$

$$
\rho_{a b}\left(v, \omega_{\alpha}, z, t\right)=P^{\prime}\left(v, \omega_{\alpha}\right) \exp (i k z-i \omega t) / 2 \mu .
$$

With the rotating-wave approximation these substitutions reduce Eqs. (1) - (4) to the new set

$$
\begin{aligned}
0= & i\left(\omega-\omega_{\alpha}-k v\right) P^{\prime}\left(v, \omega_{\alpha}\right)-\gamma P^{\prime}\left(v, \omega_{\alpha}\right) \\
& -\frac{i \mu^{2}}{\hbar} E^{\prime}\left[\rho_{a a}\left(v, \omega_{\alpha}\right)-\rho_{b b}\left(v, \omega_{\alpha}\right)\right], \\
0= & \lambda_{a}\left(v, \omega_{\alpha}\right)-\gamma_{a} \rho_{a a}\left(v, \omega_{\alpha}\right) \\
& +\frac{i}{4 \hbar}\left[E^{\prime} P^{\prime *}\left(v, \omega_{\alpha}\right)-E^{\prime *} P^{\prime}\left(v, \omega_{\alpha}\right)\right], \\
0= & \lambda_{b}\left(v, \omega_{\alpha}\right)-\gamma_{b} \rho_{b b}\left(v, \omega_{\alpha}\right)+\gamma_{a b} \rho_{a a}\left(v, \omega_{\alpha}\right) \\
& -\frac{i}{4 \hbar}\left[E^{\prime} P^{\prime *}\left(v, \omega_{\alpha}\right)-E^{\prime *} P^{\prime}\left(v, \omega_{\alpha}\right)\right] .
\end{aligned}
$$

The same substitutions reduce Eqs. (5) and (6) to the new field equation

$$
\begin{aligned}
& i \mu_{1} \sigma \omega E^{\prime}+\left(\omega^{2} \mu_{1} \epsilon_{1}-k^{2}\right) E^{\prime} \\
& =-\mu_{1} \omega^{2} \int_{0}^{\infty} \int_{-\infty}^{\infty} P^{\prime}\left(v, \omega_{\alpha}\right) d v d \omega_{\alpha} .
\end{aligned}
$$

It is sometimes convenient to introduce the new frequency parameter $\Omega=k\left(\mu_{1} \epsilon_{1}\right)^{-1 / 2}$ and the cavity lifetime $t_{c}=\epsilon_{1} / \sigma$, and then Eq. (12) can be written

$$
-\frac{E^{\prime}}{2 t_{c}}+i \frac{\omega^{2}-\Omega^{2}}{2 \omega} E^{\prime}=-\frac{i \omega}{2 \epsilon_{1}} \int_{0}^{\infty} \int_{-\infty}^{\infty} P^{\prime}\left(v, \omega_{\alpha}\right) d v d \omega_{\alpha}
$$

It is clear from the form of Eq. (13) that $\Omega$ can be interpreted as the nondispersed cavity frequency, i.e., the steady-state lasing frequency if the dispersive or real part of $P^{\prime}$ were equal to zero. It is often argued that the second term in Eq. (13) (or its equivalent in other formulations) can be reduced to $i(\omega-\Omega) E^{\prime}$ because of the closeness of the frequencies $\omega$ and $\Omega$. However, it will be shown that this approximation is not necessary and can lead to significant errors in the analysis of some lasers.

It is simplest now to require the electric-field amplitude to have the real value $E^{\prime}=E_{r}$ and to separate the polarization amplitude into its real and imaginary parts according to

$$
P^{\prime}\left(v, \omega_{\alpha}\right)=P_{r}\left(v, \omega_{\alpha}\right)+i P_{i}\left(v, \omega_{\alpha}\right) .
$$

Then Eqs. (9)-(11) and (13) can be written 


$$
\begin{aligned}
& 0=\left(\omega-\omega_{\alpha}-k v\right) P_{r}\left(v, \omega_{\alpha}\right)-\gamma P_{i}\left(v, \omega_{\alpha}\right) \\
&-\frac{\mu^{2}}{\hbar} E_{r} D\left(v, \omega_{\alpha}\right) \\
& 0=-\left(\omega-\omega_{\alpha}-k v\right) P_{i}\left(v, \omega_{\alpha}\right)-\gamma P_{r}\left(v, \omega_{\alpha}\right) \\
& 0= \lambda_{a}\left(v, \omega_{\alpha}\right)-\lambda_{b}\left(v, \omega_{\alpha}\right)-\frac{\gamma_{a}+\gamma_{a b}+\gamma_{b}}{2} D\left(v, \omega_{\alpha}\right) \\
&-\frac{\gamma_{a}+\gamma_{a b}-\gamma_{b}}{2} M\left(v, \omega_{\alpha}\right)+\frac{1}{\hbar} E_{r} P_{i}\left(v, \omega_{\alpha}\right) \\
& 0= \lambda_{a}\left(v, \omega_{\alpha}\right)+\lambda_{b}\left(v, \omega_{\alpha}\right)-\frac{\gamma_{a}-\gamma_{a b}-\gamma_{b}}{2} D\left(v, \omega_{\alpha}\right) \\
& \quad \frac{\gamma_{a}-\gamma_{a b}+\gamma_{b}}{2} M\left(v, \omega_{\alpha}\right), \\
& \frac{E_{r}}{2 t_{c}}=-\frac{\omega}{2 \epsilon_{1}} \int_{0}^{\infty} \int_{-\infty}^{\infty} P_{i}\left(v, \omega_{\alpha}\right) d v d \omega_{\alpha}, \\
& \frac{\omega^{2}-\Omega^{2}}{2 \omega} E_{r}=-\frac{\omega}{2 \epsilon_{1}} \int_{0}^{\infty} \int_{-\infty}^{\infty} P_{r}\left(v, \omega_{\alpha}\right) d v d \omega_{\alpha},
\end{aligned}
$$

where the population difference

$$
D\left(v, \omega_{\alpha}\right)=\rho_{a a}\left(v, \omega_{\alpha}\right)-\rho_{b b}\left(v, \omega_{\alpha}\right)
$$

and sum

$$
M\left(v, \omega_{\alpha}\right)=\rho_{a a}\left(v, \omega_{\alpha}\right)+\rho_{b b}\left(v, \omega_{\alpha}\right)
$$

have also been introduced.

Equations (14) and (15) can be solved for the polarizations, and the results are

$$
\begin{aligned}
& P_{r}\left(v, \omega_{\alpha}\right)=\frac{\mu^{2} D\left(v, \omega_{\alpha}\right)}{\gamma \hbar} \frac{\left[\left(\omega-\omega_{\alpha}-k v\right) / \gamma\right] E_{r}}{1+\left[\left(\omega-\omega_{\alpha}-k v\right) / \gamma\right]^{2}}, \\
& P_{i}\left(v, \omega_{\alpha}\right)=-\frac{\mu^{2} D\left(v, \omega_{\alpha}\right)}{\gamma \hbar} \frac{E_{r}}{1+\left[\left(\omega-\omega_{\alpha}-k v\right) / \gamma\right]^{2}}
\end{aligned}
$$

With these substitutions, Eq. (16) becomes

$$
\begin{aligned}
0= & \lambda_{a}\left(v, \omega_{\alpha}\right)-\lambda_{b}\left(v, \omega_{\alpha}\right)-\frac{\gamma_{a}+\gamma_{a b}+\gamma_{b}}{2} D\left(v, \omega_{\alpha}\right) \\
& -\frac{\gamma_{a}+\gamma_{a b}-\gamma_{b}}{2} M\left(v, \omega_{\alpha}\right) \\
& -\frac{\mu^{2} D\left(v, \omega_{\alpha}\right)}{\gamma \hbar^{2}} \frac{E_{r}^{2}}{1+\left[\left(\omega-\omega_{\alpha}-k v\right) / \gamma\right]^{2}} .
\end{aligned}
$$

If $\boldsymbol{M}\left(v, \omega_{\alpha}\right)$ is eliminated between Eqs. (17) and (22), one finds that the population difference can be written

$$
D\left(v, \omega_{\alpha}\right)=\frac{N\left(v, \omega_{\alpha}\right)}{1+s I\left[1+\left(\omega-\omega_{\alpha}-k v\right)^{2} / \gamma^{2}\right]^{-1}},
$$

where the unsaturated population difference is

$N\left(v, \omega_{\alpha}\right)=\left(1-\gamma_{a b} / \gamma_{b}\right) \lambda_{a}\left(v, \omega_{\alpha}\right) / \gamma_{a}-\lambda_{b}\left(v, \omega_{\alpha}\right) / \gamma_{b}$,

and the normalized intensity is

$$
s I=\frac{\mu^{2} E_{r}^{2}}{2 \hbar^{2}}\left(\frac{\gamma_{a}-\gamma_{a b}+\gamma_{b}}{2 \gamma \gamma_{a} \gamma_{b}}\right) .
$$

With the population difference from Eq. (23), the polarization components of Eqs. (20) and (21) become

$$
\begin{aligned}
& P_{r}\left(v, \omega_{\alpha}\right)=\frac{\mu^{2} N\left(v, \omega_{\alpha}\right)}{\gamma \hbar} \frac{\left[\left(\omega-\omega_{\alpha}-k v\right) / \gamma\right] E_{r}}{1+\left[\left(\omega-\omega_{\alpha}-k v\right) / \gamma\right]^{2}+s I}, \\
& P_{i}\left(v, \omega_{\alpha}\right)=-\frac{\mu^{2} N\left(v, \omega_{\alpha}\right)}{\gamma \hbar} \frac{E_{r}}{1+\left[\left(\omega-\omega_{\alpha}-k v\right) / \gamma\right]^{2}+s I} .
\end{aligned}
$$

With these results, Eqs. (18) and (19) simplify to

$$
\begin{aligned}
& \frac{1}{2 t_{\tau}}=\frac{\mu^{2} \omega}{2 \gamma \hbar \epsilon_{1}} \int_{0}^{\infty} \int_{-\infty}^{\infty} \frac{N\left(v, \omega_{\alpha}\right) d v d \omega_{\alpha}}{1+\left[\left(\omega-\omega_{\alpha}-k v\right) / \gamma\right]^{2}+s I}, \\
& \frac{\omega^{2}-\Omega^{2}}{2 \omega}=-\frac{\mu^{2} \omega}{2 \gamma \hbar \epsilon_{1}} \int_{0}^{\infty} \int_{-\infty}^{\infty} \frac{\left[\left(\omega-\omega_{\alpha}-k v\right) / \gamma\right] N\left(v, \omega_{\alpha}\right) d v d \omega_{\alpha}}{1+\left[\left(\omega-\omega_{\alpha}-k v\right) / \gamma\right]^{2}+s I} .
\end{aligned}
$$

Equations (28) and (29) can also be written

$$
\begin{aligned}
& \frac{1}{r}=\left[\int_{0}^{\infty} \int_{-\infty}^{\infty} \frac{N\left(v, \omega_{\alpha}\right) d v d \omega_{\alpha}}{1+\left[\left(\omega-\omega_{\alpha}-k v\right) / \gamma\right]^{2}+s I} / \int_{0}^{\infty} \int_{-\infty}^{\infty} \frac{N\left(v, \omega_{\alpha}\right) d v d \omega_{\alpha}}{1+\left[\left(\omega_{0}-\omega_{\alpha}-k v\right) / \gamma\right]^{2}}\right], \\
& \frac{\left(\omega^{2}-\Omega^{2}\right) t_{c}}{\omega r}=-\left[\int_{0}^{\infty} \int_{-\infty}^{\infty} \frac{\left[\left(\omega-\omega_{\alpha}-k v\right) / \gamma\right] N\left(v, \omega_{\alpha}\right) d v d \omega_{\alpha}}{1+\left[\left(\omega-\omega_{\alpha}-k v\right) / \gamma\right]^{2}+s I} / \int_{0}^{\infty} \int_{-\infty}^{\infty} \frac{N\left(v, \omega_{\alpha}\right) d v d \omega_{\alpha}}{1+\left[\left(\omega_{0}-\omega_{\alpha}-k v\right) / \gamma\right]^{2}}\right),
\end{aligned}
$$

where $r$ is a threshold parameter that references the pump rate to the value it would have at threshold $(s I=0)$ for a mode at an arbitrary frequency $\omega_{0}$ which is characteristic of the transition. Typically, $\omega_{0}$ would be chosen to be the center frequency, or the frequency of the gain maximum for asymmetric inhomogenous gain profiles. Equations (30) and (31) are a coupled set which may, in principle, be solved for the mode frequency $\omega$ and intensity $s I$. They are the basis for the following more specific frequency calculations. 


\section{HOMOGENEOUS BROADENING}

In the limit of homogeneous broadening, the atoms all have the same center frequency $\omega_{\alpha}=\omega_{0}$ and the velocity $v=0$. Then the Lorentzian terms may be removed from the integrals in Eqs. (30) and (31), and the integrated population densities cancel. Thus, the intensity and frequency are governed by the simpler formulas

$$
\begin{aligned}
& \frac{1}{r}=\frac{1}{1+\left[\left(\omega-\omega_{0}\right) / \gamma\right]^{2}+s I}, \\
& \frac{\left(\omega^{2}-\Omega^{2}\right) t_{c}}{\omega r}=-\frac{\left(\omega-\omega_{0}\right) / \gamma}{1+\left[\left(\omega-\omega_{0}\right) / \gamma\right]^{2}+s I} .
\end{aligned}
$$

The threshold parameter and intensity may be eliminated if Eq. (33) is divided by Eq. (32), and the result is

$$
\frac{\omega^{2}-\Omega^{2}}{2 \omega}=\frac{\omega_{0}-\omega}{\delta},
$$

where the decay rate parameter $\delta=2 \gamma t_{c}$ has been introduced. $^{35}$ Some insight into the meaning of Eq. (34) can be obtained before the actual length detuning formulas are considered. In particular, it is clear that Eq. (34) is a quadratic equation for the frequency $\omega$, and the positive solution is

$$
\omega_{\mathrm{ex}}=\frac{\omega_{0}+\left[\omega_{0}^{2}+\left(2 \delta+\delta^{2}\right) \Omega^{2}\right]^{1 / 2}}{2+\delta},
$$

where the subscript ex is a reminder that this result is (relatively) exact. There is also a negative-frequency solution to Eq. (34), but one finds from Eq. (32) that the oscillation threshold for the negative-frequency solution is typically much higher.

The frequency given by Eq. (35) differs from the more approximate expression that is usually given for the mode frequency in homogeneously broadened lasers. If one assumes that the lasing frequency $\omega$ is always close to the parameter $\Omega$, then Eq. (34) simplifies to

$$
\omega_{\mathrm{ap}}-\Omega=\frac{\omega_{0}-\omega_{\mathrm{ap}}}{\delta}
$$

where the subscript ap stands for approximate. The solution of Eq. (36) is

$$
\omega_{\mathrm{ap}}=\frac{\omega_{0}+\delta \Omega}{1+\delta}
$$

Equation (35) can also be reduced to Eq. (37) if the frequency difference $\omega_{0}-\Omega$ is small enough to allow an expansion of Eq. (35) to first order in this difference. The approximate formula given in Eq. (37) has long been known, and it is sometimes written in the form ${ }^{2,3}$

$$
v=\frac{v_{c} \Delta v_{h}+v_{0} \Delta v_{c}}{\Delta v_{h}+\Delta v_{c}},
$$

where $v_{c}$ is the frequency of the cavity resonance, which has a full width at half maximum of $\Delta v_{c}$, and $v_{0}$ is the center frequency of the homogeneously broadened laser medium, which has a full width at half maximum of $\Delta v_{h}$. The equivalence of Eqs. (37) and (38) follows from the re- lationships $t_{c}=\left(2 \pi \Delta \nu_{c}\right)^{-1}$ and $\gamma=\pi \Delta v_{h}$.

Since Eq. (37) (or its equivalents) is long established, it is of interest to explore the conditions under which this result represents an accurate approximation to Eq. (35). The number of variables appearing in Eqs. (35) and (37) can be reduced by introducing the normalized frequencies $\omega^{\prime}=\omega / \omega_{0}$ and $\Omega^{\prime}=\Omega / \omega_{0}$, and the results include the exact formula

$$
\omega_{\mathrm{ex}}^{\prime}=\frac{1+\left[1+\left(2 \delta+\delta^{2}\right) \Omega^{\prime 2}\right]^{1 / 2}}{2+\delta},
$$

and the approximation

$$
\omega_{\mathrm{ap}}^{\prime}=\frac{1+\delta \Omega^{\prime}}{1+\delta} \text {. }
$$

Equations (39) and (40) are plotted in Fig. 1 for various values of the parameter $\delta$. From this point of view the approximation appears more accurate in the limits of no mode pulling $(\delta=\infty)$ and strong mode pulling $(\delta=0)$, while for intermediate values of $\delta$ the frequency discrepancies are substantial. Such discrepancies are only important, however, if they occur for frequencies close enough to $\omega_{0}$ that the laser is also above the lasing threshold.

It is also important to examine the changes in lasing frequency with cavity length for the exact and approximate formulations. The oscillation phase condition requires that the round-trip phase delay must be an integral multiple of $2 \pi$. For example, if $l$ is the length of the amplifier in an otherwise empty cavity of round-trip length $L$, the phase condition can be written

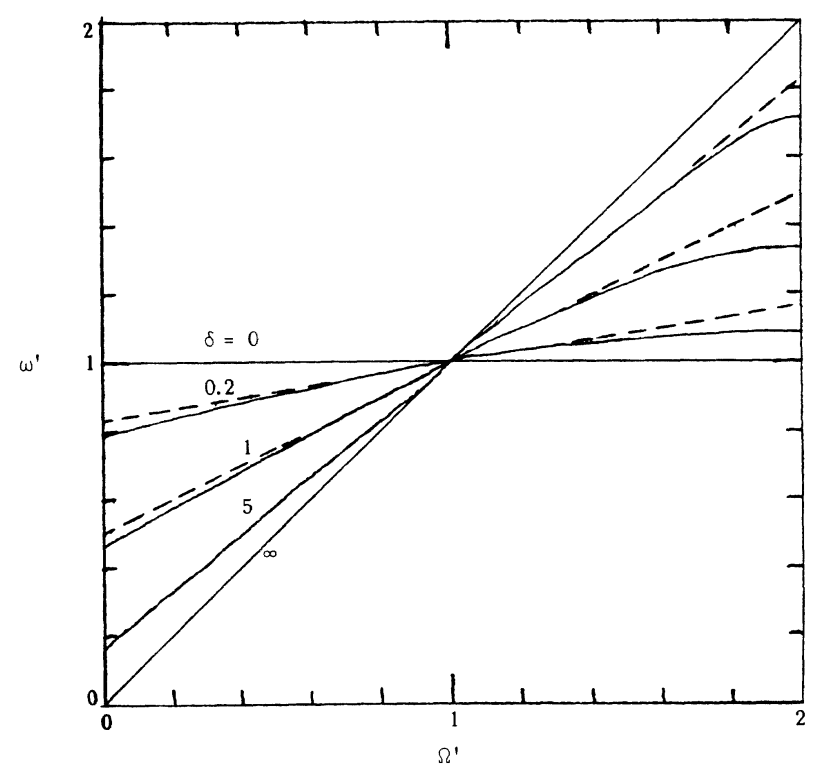

FIG. 1. Normalized lasing frequency $\omega^{\prime}=\omega / \omega_{0}$ as a function of the normalized nondispersed cavity frequency $\Omega^{\prime}=\Omega / \omega_{0}$ for various values of the decay rate parameter $\delta=2 \gamma t_{c}$. The exact values $\omega_{\text {ex }}^{\prime}$ from Eq. (39) are shown as solid lines, while the standard approximate values $\omega_{\text {ap }}^{\prime}$ from Eq. (40) are shown as dashed lines. The approximation is poorest for intermediate values of $\delta$. 


$$
\begin{aligned}
2 \pi m & =\oint k(z) d z+\phi \\
& =k l+k_{0}(L-l)+\phi \\
& =\frac{n_{1} \Omega}{c} l+\frac{\omega}{c}(L-l)+\phi,
\end{aligned}
$$

where the letter $m$ refers to the $m$ th cavity mode and the subscript 0 on the propagation constant refers to free space. The phase $\phi$ allows for possible phase changes at mirrors, and

$$
n_{1}=\left(\epsilon_{1} \mu_{1} / \epsilon_{0} \mu_{0}\right)^{1 / 2}
$$

is the nondispersed index of refraction in the amplifying medium. From Eq. (41) it follows that the empty cavity $\left(n_{1}=1, \Omega=\omega\right)$ mode frequencies are

$$
\omega_{\mathrm{em}}=\frac{2 \pi c m}{L}-\frac{c \phi}{L}
$$

and the nondispersed $\left(n_{1} \neq 1, \Omega=\omega\right)$ frequencies are

$$
\omega_{\mathrm{nd}}=\frac{\omega_{\mathrm{em}}}{1+(l / L)\left(n_{1}-1\right)} .
$$

In the general case $\Omega$ can be obtained from Eq. (41) as

$$
\begin{aligned}
\Omega & =\frac{2 \pi c m}{n_{1} l}-\frac{\omega}{n_{1}}\left(\frac{L}{l}-1\right)-\frac{c \phi}{n_{1} l} \\
& =\omega_{\mathrm{em}} \frac{L}{n_{1} l}-\frac{\omega}{n_{1}}\left(\frac{L}{l}-1\right) .
\end{aligned}
$$

When Eq. (44) is substituted into Eq. (34), one obtains a quadratic equation for the oscillation frequency:

$$
\left\{1+\frac{2}{\delta}-\left[\frac{1}{n_{1}}\left[\frac{L}{l}-1\right]\right]^{2}\right\} \omega^{2}+\left[-\frac{2 \omega_{0}}{\delta}+\frac{2 L}{n_{1} l}\left[\frac{L}{l}-1\right) \frac{\omega_{\mathrm{em}}}{n_{1}}\right] \omega-\left(\frac{L \omega_{\mathrm{em}}}{n_{1} l}\right)^{2}=0
$$

To minimize the notational burden, we will focus now on the special case of a completely filled cavity $(l=L)$ having no net phase shift at the mirrors $(\phi=0)$. In this case the positive solution of Eq. (45) can simply be written

$$
\omega_{\mathrm{ex}}^{\prime}=\frac{1+\left[1+\left(2 \delta+\delta^{2}\right) m^{2} / L^{\prime 2}\right]^{1 / 2}}{2+\delta},
$$

where the cavity length is normalized to the nondispersed wavelength in the medium for a signal at frequency $\omega_{0}$ and is denoted by $L^{\prime}$. This simplified relationship is, of course, closely related to the formula given previously as Eq. (39). The corresponding approximate result is

$$
\omega_{\mathrm{ap}}^{\prime}=\frac{1+\delta m / L^{\prime}}{1+\delta} \text {. }
$$

Equations (46) and (47) are plotted in Fig. 2 for the intermediate value $\delta=1$ and small values of $m$ and $L^{\prime}$. It is clear from the figure that the frequencies given by the two models agree exactly for operation near line center $\left(\omega^{\prime}=1\right)$ but disagree significantly for $m=0$ and also for

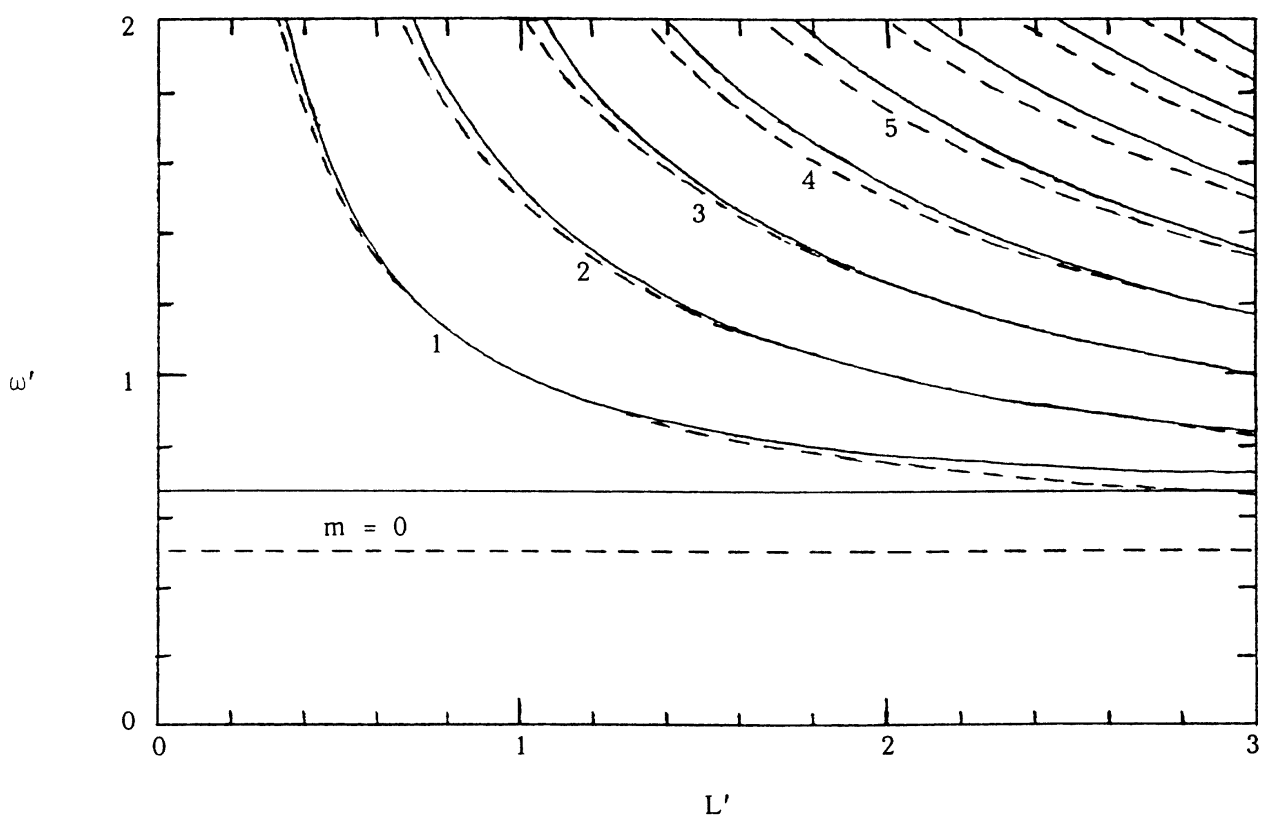

FIG. 2. Normalized lasing frequency $\omega^{\prime}=\omega / \omega_{0}$ as a function of the normalized cavity length $L^{\prime}=n_{1} \omega_{0} L / 2 \pi c=L / \lambda$ for $\delta=1$ and various values of the longitudinal mode index $m$. The exact values $\omega_{\mathrm{ex}}^{\prime}$ from Eq. (46) are shown as solid lines, while the standard approximate values $\omega_{\text {ap }}^{\prime}$ from Eq. (47) are shown as dashed lines. The approximation is poorest for $m=0$ and for large length detunings with $m>0$. 
the larger length detunings with $m>0$. For the larger values of $m$ and $L^{\prime}$ that apply to the majority of practical lasers, the corrections of interest here become less important because there would be an oscillation frequency $\omega^{\prime}$ close to unity for all detunings. The nature of the disagreement between the two models as a function of detuning can be explored in more detail by examining the second-order expansions of Eqs. (46) and (47) in terms of the length difference $\Delta L^{\prime}$ away from line center at $L^{\prime}=m$. Thus, Eq. (46) yields the expansion

$\omega_{\mathrm{ex}}^{\prime}=1-\frac{\delta}{1+\delta} \frac{\Delta L^{\prime}}{m}+\frac{\delta}{1+\delta}\left(\frac{3+4 \delta+2 \delta^{2}}{2(1+\delta)^{2}}\right)\left(\frac{\Delta L^{\prime}}{m}\right)^{2}$

while Eq. (47) gives

$$
\omega_{\mathrm{ap}}^{\prime}=1-\frac{\delta}{1+\delta} \frac{\Delta L^{\prime}}{m}+\frac{\delta}{1+\delta}\left(\frac{\Delta L^{\prime}}{m}\right)^{2} .
$$

As is also clear from Fig. 2, the approximate frequency $\omega_{\text {ap }}^{\prime}$ has the right value and slope but the wrong curvature for length detunings away from resonance. The bracketed curvature correction term in Eq. (48) has a maximum value of $\frac{3}{2}$ as $\delta$ goes to zero. The practical implications of these results are discussed in Sec. VI.

\section{INHOMOGENEOUS BROADENING}

The other important limit of the saturation formulas given in Eqs. (30) and (31) occurs when the inhomogeneous width of the function $N\left(v, \omega_{\alpha}\right)$ is much larger than the homogeneous linewidth $\Delta v_{h}=\gamma / \pi$. In this ring-laser case, the Doppler broadening due to the atomic velocities enters the analysis in the same way as other inhomogeneous broadening mechanisms, and no significant results are lost by setting $\omega_{\alpha}$ equal to $\omega_{0}$ while redefining the unsaturated population difference as

$$
N(v)=\int_{0}^{\infty} N\left(v, \omega_{\alpha}\right) d \omega_{\alpha} .
$$

Thus, Eqs. (30) and (31) simplify to

$$
\begin{aligned}
& \left.\frac{1}{r}=\iint_{-\infty}^{\infty} \frac{N(v) d v}{1+\left[\left(\omega-\omega_{0}-k v\right) / \gamma\right]^{2}+s I} / \int_{-\infty}^{\infty} \frac{N(v) d v}{1+(k v / \gamma)^{2}}\right] \\
& \frac{\left(\omega^{2}-\Omega^{2}\right) t_{c}}{\omega r}=-\left[\int_{-\infty}^{\infty} \frac{\left[\left(\omega-\omega_{0}-k v\right) / \gamma\right] N(v) d v}{1+\left[\left(\omega-\omega_{0}-k v\right) / \gamma\right]^{2}+s I} / \int_{-\infty}^{\infty} \frac{N(v) d v}{1+(k v / \gamma)^{2}}\right] .
\end{aligned}
$$

It frequently occurs that the unsaturated population difference $N(v)$ is a Gaussian function, and thus Eqs. (51) and (52) may be written explicitly

$$
\begin{aligned}
& \left.\frac{1}{r}=\iint_{-\infty}^{\infty} \frac{\exp \left(-v^{2} / u^{2}\right) d v}{1+\left[\left(\omega-\omega_{0}-k v\right) / \gamma\right]^{2}+s I} / \int_{-\infty}^{\infty} \frac{\exp \left(-v^{2} / u^{2}\right) d v}{1+(k v / \gamma)^{2}}\right), \\
& \left.\frac{\left(\omega^{2}-\Omega^{2}\right) t_{c}}{\omega r}=-\iint_{-\infty}^{\infty} \frac{\left[\left(\omega-\omega_{0}-k v\right) / \gamma\right] \exp \left(-v^{2} / u^{2}\right) d v}{1+\left[\left(\omega-\omega_{0}-k v\right) / \gamma\right]^{2}+s I} / \int_{-\infty}^{\infty} \frac{\exp \left(-v^{2} / u^{2}\right) d v}{1+(k v / \gamma)^{2}}\right),
\end{aligned}
$$

where $u$ is the most probable speed of the atoms or molecules. It is conventional to introduce new normalized variables to simplify the notation, and a possible choice includes the normalized frequency detuning $y$, velocity $V$, and natural damping ratio $\epsilon$ given by

$$
\begin{aligned}
& y=\frac{\omega-\omega_{0}}{\gamma}, \\
& V=\frac{v}{\epsilon u}=\frac{k v}{\gamma}, \\
& \epsilon=\frac{\Delta v_{h}}{\Delta v_{d}}(\ln 2)^{1 / 2} .
\end{aligned}
$$

With these substitutions Eqs. (53) and (54) simplify to

$$
\begin{aligned}
& \frac{1}{r}=\left[\int_{-\infty}^{\infty} \frac{\exp \left(-\epsilon^{2} V^{2}\right) d V}{1+(y-V)^{2}+s I} / \int_{-\infty}^{\infty} \frac{\exp \left(-\epsilon^{2} V^{2}\right) d V}{1+V^{2}}\right) \\
& \frac{\left(\omega^{2}-\Omega^{2}\right) t_{c}}{\omega r}=-\left[\int_{-\infty}^{\infty} \frac{(y-V) \exp \left(-\epsilon^{2} V^{2}\right) d V}{1+(y-V)^{2}+s I} / \int_{-\infty}^{\infty} \frac{\exp \left(-\epsilon^{2} V^{2}\right) d V}{1+V^{2}}\right) .
\end{aligned}
$$


Equations (58) and (59) are valid for arbitrary levels of homogeneous and inhomogeneous line broadening. However, special interest attaches to the frequently occurring limit in which the inhomogeneous linewidth is much larger than the homogeneous linewidth $(\epsilon<<1)$. It is shown in the Appendix that, in this limit, the leading terms in the equations may be written

$$
\begin{aligned}
& \frac{1}{r}=\frac{\exp \left(-x^{2}\right)}{(1+s I)^{1 / 2}}, \\
& \frac{\left(\omega^{2}-\Omega^{2}\right) t_{c}}{\omega r}=-\frac{2}{\pi^{1 / 2}} F(x),
\end{aligned}
$$

where $x=\epsilon y$, and $F(x)$ is the Gaussian dispersion function. This function has also been called Dawson's integral and is given by ${ }^{36}$

$$
F(x)=\exp \left(-x^{2}\right) \int_{0}^{x} \exp \left(t^{2}\right) d t
$$

Equation (61) does not depend on the intensity, so this equation may be solved directly (in principle) for the oscillation frequency without reference to Eq. (60). The resulting frequency would be substituted into Eq. (60) to obtain the intensity. If Eq. (44) is used to eliminate $\Omega$, Eq. (61) completely determines the oscillation frequency. Numerical or graphical techniques are required for the solution of this equation.

Some of the mode-pulling results for inhomogeneously broadened lasers are similar to the corresponding results for homogeneous broadening, and detailed solutions are omitted here. For frequencies close to line center, the function $F(x)$ given in Eq. (62) may be replaced by $x=\epsilon\left(\omega-\omega_{0}\right) / \gamma$, and Eq. (61) becomes

$$
\frac{\omega^{2}-\Omega^{2}}{2 \omega}=\frac{\omega_{0}-\omega}{\delta} \frac{2 \epsilon r}{\pi^{1 / 2}} \text {. }
$$

This result is of the same form as Eq. (34) for homogeneous broadening if the parameter $\delta$ in Eq. (34) is replaced by $\delta^{\prime}=\pi^{1 / 2} \delta / 2 \epsilon r$.

It may also be noted that, for narrow bandwidth lasers, Eq. (61) reduces to known results. If $\omega$ is close to $\Omega, \mathbf{E q}$. (61) can be approximated by

$$
\omega-\Omega=-\frac{r}{\pi^{1 / 2} t_{c}} F(x) .
$$

With Eq. (44) this is

$$
\omega_{\mathrm{em}}-\omega\left[l+\frac{l}{L}\left(n_{1}-1\right)\right]=\frac{n_{1} l}{L} \frac{r}{\pi^{1 / 2} t_{c}} F(x) .
$$

In gas lasers the background index of refraction $n_{1}$ is near unity, and in this case Eq. (65) reduces to Eq. (6) of Ref. 5.

\section{STANDING-WAVE LASERS}

In the previous section, Eqs. (1)-(6) were applied to the problem of calculating the mode frequency of ring lasers. The same equations also govern standing-wave lasers, when one adopts appropriate forms for the electric-field and off-diagonal density-matrix elements. In particular, Eqs. (7) and (8) should be replaced by

$$
\begin{aligned}
& E(z, t)=\frac{1}{2} E^{\prime} \sin (k z) \exp (-i \omega t)+\text { c.c. }, \\
& \rho_{a b}\left(v, \omega_{\alpha}, z, t\right)=P^{\prime}\left(v, \omega_{\alpha}, z\right) \exp (-i \omega t) / 2 \mu .
\end{aligned}
$$

A detailed derivation of the intensity and frequency equations for a slightly simpler model of the standing-wave laser $\left(\gamma_{a b}=0\right)$ has been given previously in the appendix of Ref. 27. For brevity, only the more general resulting formulas will be given here for the three important cases of homogeneous broadening, non-Doppler inhomogeneous broadening, and Doppler inhomogeneous broadening.

In a non-Doppler inhomogeneously broadened standing-wave laser, the intensity and field equations become

$$
\begin{gathered}
\frac{1}{r}=\frac{2 \int_{0}^{\infty} \frac{N\left(\omega_{\alpha}\right)}{1+\left[\left(\omega-\omega_{\alpha}\right) / \gamma\right]^{2}}\left[1+\frac{4 s I}{1+\left[\left(\omega-\omega_{\alpha}\right) / \gamma\right]^{2}}+\left[1+\frac{4 s I}{1+\left[\left(\omega-\omega_{\alpha}\right) / \gamma\right]^{2}}\right]^{1 / 2}\right]^{-1} d \omega_{\alpha}}{\int_{0}^{\infty} \frac{N\left(\omega_{\alpha}\right) d \omega_{\alpha}}{1+\left[\left(\omega_{0}-\omega_{\alpha}\right) / \gamma\right]^{2}}}, \\
\frac{\left(\omega^{2}-\Omega^{2}\right) t_{c}}{\omega r}=-\frac{2 \int_{0}^{\infty} \frac{\left[\left(\omega-\omega_{\alpha}\right) / \gamma\right] N\left(\omega_{\alpha}\right)}{1+\left[\left(\omega-\omega_{\alpha}\right) / \gamma\right]^{2}}\left[1+\frac{4 s I}{1+\left[\left(\omega-\omega_{\alpha}\right) / \gamma\right]^{2}}+\left[1+\frac{4 s I}{1+\left[\left(\omega-\omega_{\alpha}\right) / \gamma\right]^{2}}\right]^{1 / 2}\right]^{-1} d \omega_{\alpha}}{\int_{0}^{\infty} \frac{N\left(\omega_{\alpha}\right) d \omega_{\alpha}}{1+\left[\left(\omega_{0}-\omega_{\alpha}\right) / \gamma\right]^{2}}},
\end{gathered}
$$

where the normalized intensity is

$$
s I=\frac{\mu^{2} E_{r}^{2}}{8 \hbar^{2}} \frac{\gamma_{a}-\gamma_{a b}+\gamma_{b}}{\gamma \gamma_{a} \gamma_{b}} .
$$

These equations may be solved numerically for the intensity $s I$ and frequency $\omega$.

In a homogeneously broadened laser $\left(\omega_{\alpha}=\omega_{0}\right)$, Eqs. (68) and (69) reduce to 


$$
\begin{aligned}
& \frac{1}{r}=\frac{2}{1+\left[\left(\omega-\omega_{0}\right) / \gamma\right]^{2}}\left[1+\frac{4 s I}{1+\left[\left(\omega-\omega_{0}\right) / \gamma\right]^{2}}+\left[1+\frac{4 s I}{1+\left[\left(\omega-\omega_{0}\right) / \gamma\right]^{2}}\right]^{1 / 2}\right]^{-1} \\
& \frac{\left(\omega^{2}-\Omega^{2}\right) t_{c}}{\omega r}=-\frac{2\left[\left(\omega-\omega_{0}\right) / \gamma\right]}{1+\left[\left(\omega-\omega_{0}\right) / \gamma\right]^{2}}\left[1+\frac{4 s I}{1+\left[\left(\omega-\omega_{0}\right) / \gamma\right]^{2}}+\left[1+\frac{4 s I}{1+\left[\left(\omega-\omega_{0}\right) / \gamma\right]^{2}}\right]^{1 / 2}\right]^{-1}
\end{aligned}
$$

In this case, both the frequency and intensity can be obtained analytically. In particular, dividing Eq. (72) by Eq. (71) yields Eq. (34). Thus, the oscillation frequency characteristics of a homogeneously broadened laser are the same for a one-dimensional ring resonator as for a standing-wave resonator. After the frequency is determined, the intensity can be obtained analytically by solving Eq. (71).

In a Doppler inhomogeneously broadened standing-wave laser, the intensity and frequency are governed by the equations

$$
\begin{aligned}
& \frac{1}{r}=\left[\int_{-\infty}^{\infty} \frac{N(v) W_{r}(v)}{1+2 W_{r}(v) s I} d V / \int_{-\infty}^{\infty} N(v) \alpha_{\text {or }}(v) d v\right], \\
& \frac{\left(\omega^{2}-\Omega^{2}\right) t_{c}}{\omega r}=-\left[\int_{-\infty}^{\infty} N(v) \operatorname{Re}\left[\frac{\omega-\omega_{0}}{\gamma+i k v} \frac{W(v)}{1+2 W_{r}(v) s I}\right] d v / \int_{-\infty}^{\infty} N(v) \alpha_{\text {or }}(v) d v\right],
\end{aligned}
$$

where $W_{r}(v)$ is the real part of the continued fraction

$$
W(v)=\frac{\alpha_{0}(v)}{1+\frac{\alpha_{0}(v) \beta_{1}(v) s I}{1+\frac{\alpha_{1}(v) \beta_{1}(v) s I}{1+\frac{\alpha_{1}(v) \beta_{2}(v) s I}{1+\cdots}}}} .
$$

In this expression $\alpha_{j}(v)$ and $\beta_{j}(v)$ are defined by

$$
\begin{aligned}
\alpha_{j}(v)= & \frac{\gamma / 2}{(2 j+1) i k v+i\left(\omega-\omega_{0}\right)+\gamma} \\
& +\frac{\gamma / 2}{(2 j+1) i k v-i\left(\omega-\omega_{0}\right)+\gamma}, \\
\beta_{j}(v)= & \frac{\gamma_{a} \gamma_{b}}{\gamma_{a}-\gamma_{a b}+\gamma_{b}} \\
& \times\left[\frac{(4 j) i k v+\gamma_{a}-\gamma_{a b}+\gamma_{b}}{\left[(2 j) i k v+\gamma_{a}\right]\left[(2 j) i k v+\gamma_{b}\right]}\right] .
\end{aligned}
$$

The function $\alpha_{\text {or }}(v)$ appearing in Eqs. (73) and (74) is given by the real part of Eq. (76) with the subscript $j$ set equal to zero. Equations (73) and (74) may be solved numerically for the intensity and frequency, and in some limits of the decay rates further simplifications are also possible.

\section{DISCUSSION}

In the preceding sections mode-pulling formulas have been derived for a variety of simple laser configurations; and a fundamental field-equation approximation has been examined and avoided. While there are immediate philosophical arguments for eliminating any unnecessary approximation, it is also appropriate to consider the conditions under which the more accurate frequency equations might provide significantly better agreement with experimental data. It is evident already from the graphical re- sults in Sec. III that, in a typical narrow bandwidth $\left(\omega_{0} \gg \gamma\right)$ or long $(L \gg \lambda)$ laser cavity, the lasing mode will be so near to line center that the correction to the standard formulas would be negligibly small. Therefore, attention will instead be focused on lasers which are very short and have large tuning ranges.

The fundamental requirement for a short laser is that the amplifying medium must have very high incremental gain, and one thinks immediately of dye lasers, semiconductor lasers, and a few other media that have been operated in optically pumped platelet configurations. The demonstration of single-atom lasers also provides evidence of potentially very high incremental gain. ${ }^{37}$ Conventional semiconductor lasers have the further desirable feature of combining their high gain with a moderately wide tuning range, but they are surpassed in this respect by dye laser systems. Perhaps the most interesting lasers in this context are the recently developed so-called vertical-cavity quantum-well lasers. In these lasers the optical signal propagates across one or more thin layers of very high-gain semiconducting material while reflecting between integrally fabricated mirrors. Cavity lengths of only a few wavelengths are becoming common, and a gain medium as short at $80 \AA$ has been successfully employed. ${ }^{38}$ The tuning range of quantumwell lasers is also very large, and values beyond $10 \%$ have been reported. ${ }^{39}$ The requirement of small $\delta=2 \gamma t_{c}$ implies very small values of $t_{c}$; but, with such high-gain media and short cavities, femtosecond lifetimes should be possible. In other words, there are no fundamental obstacles preventing the operation of lasers under conditions where the standard formulas would be substantially in error, and for some systems such conditions may already have been attained. The approximation made above that the gain and loss are uniformly distributed over the length of the laser cavity would not necessarily be valid in such lasers, and it will be of interest to generalize this treatment to include such spatial gain and loss variations.

While the analysis here was developed for the simplest 
possible single-mode laser oscillators, the implications are much broader because the approximation under consideration has been so widely employed. Thus, in the longer and more easily fabricated multimode lasers, mode spacings and mode-locking behavior might be significantly altered. Also, the closely related derivative approximation has been widely used in studies of the time-dependent or spatially dependent electromagnetic fields in lasers and other nonlinear optical systems, as the second-order wave equation is reduced to a first-order equation in the time or distance variables. The accuracy of such methods in interpreting, for example, the formation or measurement of fsec pulsations, is not selfevident. Basically, the optical fields in some high-gain media now vary substantially in a distance of one wavelength or a time of one optical cycle, and fundamental approximations that have long been taken for granted can no longer be accepted without careful verification.

\section{ACKNOWLEDGMENTS}

This work was supported in part by the National Science Foundation and by Tektronix, Inc. The author also expresses his appreciation to W. J. Sandle, R. J. Ballagh, D. M. Warrington, and other members of the Department of Physics at the University of Otago for valuable discussions and hospitality during his sabbatical visit.

\section{APPENDIX: GAIN AND DISPERSION INTEGRALS}

The purpose of this appendix is to show how the Gaussian gain and dispersion integrals given as Eqs. (58) and (59) above can be simplified near the limit of inhomogeneous broadening. The results disagree with a standard reference on this subject. For clarity Eqs. (58) and (59) are reproduced here:

$$
\begin{aligned}
& \frac{1}{r}=\left(\int_{-\infty}^{\infty} \frac{\exp \left(-\epsilon^{2} V^{2}\right) d V}{1+(y-V)^{2}+s I} / \int_{-\infty}^{\infty} \frac{\exp \left(-\epsilon^{2} V^{2}\right) d V}{1+V^{2}}\right), \\
& \frac{\left(\omega^{2}-\Omega^{2}\right) t_{c}}{\omega r}=-\left(\int_{-\infty}^{\infty} \frac{(y-V) \exp \left(-\epsilon^{2} V^{2}\right) d V}{1+(y-V)^{2}+s I} / \int_{-\infty}^{\infty} \frac{\exp \left(-\epsilon^{2} V^{2}\right) d V}{1+V^{2}}\right) .
\end{aligned}
$$

Specifically, the leading two terms in Eqs. (A1) and (A2) will be derived for an expansion in powers of the natural damping ratio $\epsilon$.

The first integral to consider is the normalization integral which appears in the denominators of Eqs. (A1) and (A2) and which we will identify as $I_{1}$ given by

$$
I_{1}=\int_{-\infty}^{\infty} \frac{\exp \left(-\epsilon^{2} V^{2}\right) d V}{1+V^{2}}
$$

For small values of $\epsilon$ it is clear that the numerator is a much broader function of $V$ than the denominator, and the desired expansion could be obtained simply by expanding the numerator in a power series prior to performing the integration. In this case we are only interest- ed in terms up to first order in $\epsilon$, so the result is only the one term

$$
I_{1}=\int_{-\infty}^{\infty} \frac{d V}{1+V^{2}}=\pi .
$$

The second integral of interest appears in the numerator of Eq. (A1) and will be identified here as $I_{2}$ :

$$
I_{2}=\int_{-\infty}^{\infty} \frac{\exp \left(-\epsilon^{2} V^{2}\right) d V}{1+(y-V)^{2}+s I} .
$$

It is helpful to introduce the new parameters $x=\epsilon y$ and $p=\epsilon(V-y)$, and then with some simple but tedious manipulations Eq. (A5) can be written to first order in $\epsilon$

$$
\begin{aligned}
& I_{2}=\epsilon \exp \left(-x^{2}\right) \int_{-\infty}^{\infty} \frac{\exp \left(-2 x p-p^{2}\right) d p}{\epsilon^{2}(1+s I)+p^{2}} \\
& \left.=\epsilon \exp \left(-x^{2}\right)\left[\mid-2 \int_{0}^{x} \int_{-\infty}^{\infty} \frac{p \exp \left(-2 t p-p^{2}\right) d p d t}{\epsilon^{2}(1+s I)+p^{2}}\right]+\left[\int_{-\infty}^{\infty} \frac{\exp \left(-2 t p-p^{2}\right) d p}{\epsilon^{2}(1+s I)+p^{2}}\right]_{t=0}\right] \\
& =\epsilon \exp \left(-x^{2}\right)\left\{-2 \int_{0}^{x}\left[\mid-2 \int_{0}^{t} \int_{-\infty}^{\infty} \frac{p^{2} \exp \left(-2 q p-p^{2}\right) d p d q}{\epsilon^{2}(1+s I)+p^{2}}\right]\right. \\
& \left.\left.\quad+\left[\int_{-\infty}^{\infty} \frac{p \exp \left(-2 q p-p^{2}\right) d p}{\epsilon^{2}(1+s I)+p^{2}}\right]_{q=0}\right] d t+\int_{-\infty}^{\infty} \frac{\exp \left(-p^{2}\right) d p}{\epsilon^{2}(1+s I)+p^{2}}\right\} \\
& =\epsilon \exp \left(-x^{2}\right)\left[-2 \int_{0}^{x}\left[-2 \int_{0}^{t} \exp \left(q^{2}\right) \int_{-\infty}^{\infty} \frac{p^{2} \exp \left[-(q+p)^{2}\right] d p d q}{\epsilon^{2}(1+s I)+p^{2}}\right.\right. \\
& \left.\left.\quad+\int_{-\infty}^{\infty} \frac{p \exp \left(-p^{2}\right) d p}{\epsilon^{2}(1+s I)+p^{2}}\right] d t+\int_{-\infty}^{\infty} \frac{d p}{\epsilon^{2}(1+s I)+p^{2}}\right]
\end{aligned}
$$




$$
\begin{aligned}
& =\epsilon \exp \left(-x^{2}\right)\left\{-2 \int_{0}^{x}\left[-2 \int_{0}^{t} \exp \left(q^{2}\right) \int_{-\infty}^{\infty}\left(1-\frac{\epsilon^{2}(1+s I)}{\epsilon^{2}(1+s I)+p^{2}}\right)\right.\right. \\
& \left.\left.\times \exp \left[-(q+p)^{2}\right] d p d q\right] d t+\frac{\pi}{\epsilon(1+s I)^{1 / 2}}\right\} \\
& =\frac{\pi \exp \left(-x^{2}\right)}{(1+s I)^{1 / 2}}+4 \epsilon \exp \left(-x^{2}\right) \int_{0}^{x} \int_{0}^{t} \exp \left(q^{2}\right) \int_{-\infty}^{\infty} \exp \left[-(q+p)^{2}\right] d p d q d t \\
& =\frac{\pi \exp \left(-x^{2}\right)}{(1+s I)^{1 / 2}}+4 \pi^{1 / 2} \epsilon \exp \left(-x^{2}\right) \int_{0}^{x} \int_{0}^{t} \exp \left(q^{2}\right) d q d t \\
& =\frac{\pi \exp \left(-x^{2}\right)}{(1+s I)^{1 / 2}}+4 \pi^{1 / 2} \epsilon \exp \left(-x^{2}\right) \int_{0}^{x} \int_{q}^{x} \exp \left(q^{2}\right) d t d q \\
& =\frac{\pi \exp \left(-x^{2}\right)}{(1+s I)^{1 / 2}}-4 \pi^{1 / 2} \epsilon \exp \left(-x^{2}\right) \int_{0}^{x}(q-x) \exp \left(q^{2}\right) d q \\
& =\frac{\pi \exp \left(-x^{2}\right)}{(1+s I)^{1 / 2}}-2 \pi^{1 / 2} \epsilon\left[\exp \left(-x^{2}\right) \int_{0}^{x^{2}} \exp \left(q^{2}\right) d q^{2}-2 x F(x)\right) \\
& =\frac{\pi \exp \left(-x^{2}\right)}{(1+s I)^{1 / 2}}-2 \pi^{1 / 2} \epsilon\left[1-\exp \left(-x^{2}\right)-2 x F(x)\right],
\end{aligned}
$$

where higher-order terms in $\epsilon$ have been dropped as the calculation progressed, and the Gaussian dispersion function is defined as ${ }^{36}$

$$
F(x)=\exp \left(-x^{2}\right) \int_{0}^{x} \exp \left(t^{2}\right) d t
$$

The third integral of interest appears in the numerator of Eq. (A2) and will be identified here as $I_{3}$ :

$$
\begin{aligned}
I_{3} & =-\int_{-\infty}^{\infty} \frac{(y-V) \exp \left(-\epsilon^{2} V^{2}\right) d V}{1+(y-V)^{2}+s I} \\
& =\exp \left(-x^{2}\right) \int_{-\infty}^{\infty} \frac{p \exp \left(-2 x p-p^{2}\right) d p}{\epsilon^{2}(1+s I)+p^{2}} \\
& \left.=\exp \left(-x^{2}\right)\left[\mid-2 \int_{0}^{x} \int_{-\infty}^{\infty} \frac{p^{2} \exp \left(-2 t p-p^{2}\right) d p d t}{\epsilon^{2}(1+s I)+p^{2}}\right]+\left[\int_{-\infty}^{\infty} \frac{p \exp \left(-2 t p-p^{2}\right) d p}{\epsilon^{2}(1+s I)+p^{2}}\right]_{t=0}\right] \\
& =\exp \left(-x^{2}\right)\left[-2 \int_{0}^{x} \exp \left(t^{2}\right) \int_{-\infty}^{\infty} \frac{p^{2} \exp \left[-(t+p)^{2}\right] d p d t}{\epsilon^{2}(1+s I)+p^{2}}+\int_{-\infty}^{\infty} \frac{p \exp \left(-p^{2}\right) d p}{\epsilon^{2}(1+s I)+p^{2}}\right] \\
& =-2 \exp \left(-x^{2}\right) \int_{0}^{x} \exp \left(t^{2}\right) \int_{-\infty}^{\infty}\left[1-\frac{\epsilon^{2}(1+s I)}{\epsilon^{2}(1+s I)+p^{2}}\right] \exp \left[-(t+p)^{2}\right] d p d t \\
& =-2 \exp \left(-x^{2}\right) \int_{0}^{x} \exp \left(t^{2}\right)\left[\int_{-\infty}^{\infty} \exp \left[-(t+p)^{2}\right] d p-\epsilon^{2}(1+s I) \int_{-\infty}^{\infty} \frac{\exp \left[-(t+p)^{2}\right] d p}{\epsilon^{2}(1+s I)+p^{2}}\right] d t \\
& =-2 \exp \left(-x^{2}\right) \int_{0}^{x} \exp \left(t^{2}\right)\left[\pi^{1 / 2}-\pi \epsilon(1+s I)^{1 / 2} \exp \left(-t^{2}\right)\right] d t \\
& =-2 \exp \left(-x^{2}\right) \int_{0}^{x} \exp \left(t^{2}\right)\left[\pi^{1 / 2}-\epsilon^{2}(1+s I) \exp \left(-t^{2}\right) \int_{-\infty}^{\infty} \frac{d p}{\epsilon^{2}(1+s I)+p^{2}}\right] d t
\end{aligned}
$$


Using the three integrals derived above, Eqs. (A1) and (A2) can be written

$$
\begin{gathered}
\frac{1}{r}=\frac{\exp \left(-x^{2}\right)}{(1+s I)^{1 / 2}}-\frac{2 \epsilon}{\pi^{1 / 2}}\left[1-\exp \left(-x^{2}\right)-2 x F(x)\right] \\
\begin{aligned}
\frac{\left(\omega^{2}-\Omega^{2}\right) t_{c}}{\omega r} & =-\frac{2}{\pi^{1 / 2}} F(x) \\
& +2 \epsilon(1+s I)^{1 / 2} x \exp \left(-x^{2}\right)
\end{aligned}
\end{gathered}
$$

It is clear from Eq. (A10) that, for small values of $\epsilon$, the oscillation frequency is independent of the intensity. This independence occurs because, in an inhomogeneously broadened medium, the saturation correction to the dispersion is essentially an antisymmetric function centered at the frequency of the saturating field. The righthand sides of Eqs. (A9) and (A10) can almost be harmonized with the results quoted in Eqs. (43) and (44) of Ref. 13, except for the presence here of the bracketed exponential in Eq. (A9). Without that exponential, the linear term in $\epsilon$ would not vanish at line center $(x=y=0)$, which, from Eq. (A1), it clearly must do. The leading terms in Eqs. (A9) and (A10) appear above in Eqs. (60) and (61). Higher-order terms in $\epsilon$ can be obtained by similar methods.
For many purposes one is not interested in quantitative accuracy for large values of the detuning $x$, since the laser then would likely be below the lasing threshold. It is useful to perform a power-series expansion of the integrand in Eq. (A7), and then one obtains

$$
F(x)=\left[x+\frac{x^{3}}{3}+\frac{x^{5}}{5 \times 2 !}+\frac{x^{7}}{7 \times 3 !}+\cdots\right) \exp \left(-x^{2}\right) .
$$

With this substitution the integral terms in Eqs. (A9) and (A10) are straightforward to evaluate numerically. Keeping only the leading terms in the expansion yields the following sort of explicit approximation:

$$
\begin{aligned}
& \frac{1}{r}=\frac{\exp \left(-x^{2}\right)}{(1+s I)^{1 / 2}} \\
& -\frac{2 \epsilon}{\pi^{1 / 2}}\left[1-\left(1+2 x^{2}+\frac{2 x^{4}}{3}\right) \exp \left(-x^{2}\right)\right] \text {, } \\
& \frac{\left(\omega^{2}-\Omega^{2}\right) t_{c}}{\omega r}=-\frac{2 x}{\pi^{1 / 2}}\left(1+\frac{x^{2}}{3}+\frac{x^{4}}{10}\right) \exp \left(-x^{2}\right) \\
& +2 \epsilon(1+s I)^{1 / 2} x \exp \left(-x^{2}\right) \text {. }
\end{aligned}
$$

"Permanant address: Department of Electrical Engineering, Portland State University, Portland, OR 97207-0751.

${ }^{1}$ A. L. Schawlow and C. H. Townes, Phys. Rev. 112, 1940 (1958).

${ }^{2}$ C. H. Townes, in Advances in Quantum Electronics, edited by J. R. Singer (Columbia University Press, New York, 1961), pp. 3-11.

${ }^{3}$ W. R. Bennett, Jr., Phys. Rev. 126, 580 (1962).

${ }^{4}$ W. E. Lamb, Jr., Phys. Rev. 134, A1429 (1964).

${ }^{5}$ L. W. Casperson and A. Yariv, Appl. Phys. Lett. 17, 259 (1970).

${ }^{6}$ A. Javan, E. A. Ballik, and W. L. Bond, J. Opt. Soc. Am. 52, 96 (1962).

${ }^{7}$ W. R. Bennett, Jr., S. F. Jacobs, J. T. LaTourrette, and P. Rabinowitz, Appl. Phys. Lett. 5, 56 (1964).

${ }^{8}$ R. A. McFarlane, Phys. Rev. 135, A543 (1964).

${ }^{9}$ P. T. Bolwijn, Appl. Phys. Lett. 6, 203 (1965).

${ }^{10}$ H. S. Boyne, M. M. Birky, and W. G. Schweitzer, Jr., Appl. Phys. Lett. 7, 62 (1965).

${ }^{11}$ F. Aronowitz, Phys. Rev. 139, A635 (1965).

${ }^{12}$ T. J. Hutchings, J. Winocur, R. H. Durrett, E. D. Jacobs, and W. L. Zingery, Phys. Rev. 152, 467 (1966).

${ }^{13}$ D. H. Close, Phys. Rev. 153, 360 (1967).

${ }^{14}$ C. S. Liu, J. T. Verdeyen, and B. E. Cherrington, IEEE J. Quantum Electron. QE-4, 388 (1968).

${ }^{15}$ A. Dienes, Phys. Rev. 174, 400 (1968).

${ }^{16}$ A. Dienes, Phys. Rev. 174, 414 (1968).

${ }^{17}$ H. Greenstein, Phys. Rev. 175, 438 (1968).

${ }^{18}$ B. K. Garside, IEEE J. Quantum Electron. QE-5, 97 (1969).

${ }^{19}$ C. Whitney, Phys. Rev. 181, 535 (1969).

${ }^{20}$ C. S. Liu, B. E. Cherrington, and J. T. Verdeyen, J. Appl. Phys. 40, 3556 (1969).

${ }^{21}$ N. L. Balazs and I. Tobias, Philos. Trans. R. Soc. London,
Ser. A 264, 1 (1969).

${ }^{22}$ B. J. Feldman and M. S. Feld, Phys. Rev. A 1, 1375 (1970).

${ }^{23}$ E. I. Tsetsegova, Opt. Spectrosc. 31, 172 (1971).

${ }^{24}$ P. W. Smith and P. J. Maloney, Appl. Phys. Lett. 22, 667 (1973).

${ }^{25}$ L. Lynds, M. Foster, L. Boedeker, A. Angelbeck, and G. Wisner, IEEE J. Quantum Electron. QE-10, 702 (1974).

${ }^{26}$ A. S. Bulygin and V. P. Kapralov, Opt. Spectrosc. 40, 91 (1976).

${ }^{27}$ L. W. Casperson, Appl. Opt. 19, 422 (1980).

${ }^{28}$ P. W. Milonni, Appl. Opt. 20, 1571 (1981).

${ }^{29}$ L. A. Schlie, J. Opt. Soc. Am. 71, 1080 (1981).

${ }^{30}$ L. Lynds, A. W. Angelbeck, and B. Burdick, Opt. Commun. 47, 335 (1983).

${ }^{31}$ R. S. Gioggia and N. B. Abraham, Phys. Rev. A 29, 1304 (1984).

${ }^{32}$ W. Jamroz, D. Hugon, T. B. Cave, A. Guest, and A. D. May, Appl. Opt. 23, 2906 (1984).

${ }^{33}$ S. C. Mehendal and R. G. Harrison, Phys. Rev. A 34, 1613 (1986).

${ }^{34}$ T. Kariya, T. Saito, Y. Kiwamoto, H. Gotoh, and S. Miyoshi, Jpn. J. Appl. Phys. 25, 654 (1986).

${ }^{35}$ L. W. Casperson, Phys. Rev. A 23, 248 (1981)

${ }^{36}$ Handbook of Mathematical Functions, Natl. Bur. Stand. Appl. Math Ser. No. 55, edited by M. Abramowitz and I. A. Stegun (U.S. GPO, Washington, D.C., 1964), pp. 297-304.

${ }^{37}$ D. Meschede, H. Walther, and G. Mueller, Phys. Rev. Lett. 54, 551 (1985).

${ }^{38}$ J. L. Jewell, K. F. Huang, K. Tai, Y. H. Lee, R. J. Fischer, S. L. McCall, and A. Y. Cho, Appl. Phys. Lett. 55, 424 (1989).

${ }^{39}$ M. Mittelstein, D. Mehuys, A. Yariv, J. E. Ungar, and R. Sarfaty, Appl. Phys. Lett. 54, 1092 (1989). 